\title{
Politique
}

Politique

Pierre Hamel, Jean-François Léonard et Robert Mayer, Les

mobilisations populaires urbaines, Montréal, Nouvelle

optique, 1982

Gisèle Ampleman, Gérald Doré, Lorraine Gaudreau, Claude

Larose, Louise Leboeuf, Denise Ventelou. Pratiques de

conscientisation; expériences d'éducation populaire au

Québec, Montréal, Nouvelle opitque, 1983

\section{Caroline Andrew}

Numéro 5, hiver 1984

Femmes et pouvoir

URI : https://id.erudit.org/iderudit/040457ar

DOI : https://doi.org/10.7202/040457ar

Aller au sommaire du numéro

Éditeur(s)

Société québécoise de science politique

ISSN

0711-608X (imprimé)

1918-6584 (numérique)

Découvrir la revue

Citer ce compte rendu

Andrew, C. (1984). Compte rendu de [Pierre Hamel, Jean-François Léonard et

Robert Mayer, Les mobilisations populaires urbaines, Montréal, Nouvelle optique, 1982 / Gisèle Ampleman, Gérald Doré, Lorraine Gaudreau, Claude Larose, Louise Leboeuf, Denise Ventelou. Pratiques de conscientisation; expériences d'éducation populaire au Québec, Montréal, Nouvelle opitque, 1983]. Politique, (5), 162-165. https://doi.org/10.7202/040457ar d'utilisation que vous pouvez consulter en ligne. 
Pierre Hamel, Jean-François Léonard et Robert Mayer, Les mobilisations populaires urbaines, Montréal, Nouvelle optique, 1982.

Gisèle Ampleman, Gérald Doré, Lorraine Gaudreau, Claude Larose, Louise Leboeuf, Denise Ventelou. Pratiques de conscientisation; expériences d'éducation populaire au Québec, Montréal, Nouvelle optique, 1983.

Il est fascinant de lire ces deux livres ensemble car, de façon très générale, le sujet est le même dans les deux. En même temps le traitement, la perspective, les questions posées sont tout à fait différents et donc nous avons le plaisir de tenter de reconstruire la réalité un peu comme si nous faisions un casse-tête.

Les similarités - les deux livres analysent les groupes populaires au Québec. En plus la période étudiée est assez semblable- 
Hamel utilise la période 1960-80 et l'autre, les années 70 . Certaines expériences reviennent dans les deux - notamment touchant le Regroupement des organisateurs communautaires du Québec (Hamel, pp. 319-340, Ampleman, pp. 101-130).

Mais les différences sont aussi très importantes. Le livre d'Hamel est un recueil de textes, surtout des groupes populaires, mais également des analystes de ces groupes. Le but du livre est de fournir un matériel qui permet une évaluation de la portée politique des actions des groupes. La question centrale: qu'estce que ça a changé pour la société politique?

Le livre d'Ampleman s'organise autour des idées de l'éducation populaire et de la culture ouvrière - comment on peut faire de l'éducation populaire en tenant compte de la culture ouvrière. ( «Nous parlerons donc de pratiques enracinées dans des démarches actives de connaissance des traits culturels de la classe populaire, déployant de multiples outils pédagogiques pour retourner à des groupes leur situation sous forme de défi à relever dans l'action» - p. 11). Leur question centrale: qu'est-ce que ça a changé pour les gens impliqués dans ces projets?

À cette différence centrale se greffent d'autres différences secondaires. Le livre d'Hamel parle surtout d'expériences montréalaises, celui d'Ampleman surtout de Québec. Le ton du livre d'Hamel est celui de l'analyse extérieure tandis que le livre d'Ampleman est écrit d'un ton personnel, engagé, passionné.

Dans les deux cas, par la nature des livres, l'argument principal des auteurs se dégage surtout de l'organisation du matériel. Hamel a divisé les textes en quatre sections, en commençant par une section sur l'évolution des groupes pendant la période 1960-80, (la diversité des fronts de lutte, l'évolution du débat sur l'organisation interne des groupes). Ensuite les auteurs abordent la question de l'État et les liens groupes-État et organisent les textes de façon à démontrer la forte intervention de l'État et la menace de l'étatisation qui pèse sur les groupes populaires. 
Ensuite les auteurs posent la question du rôle des intellectuels dans les groupes populaires. Question qui permet à ceux-ci de reprendre le fils de l'évolution interne et aussi celui des rapports État/groupes et ainsi de situer les animateurs/intellectuels par rapport aux groupes dans une double perspective interne et externe. La dernière section sur la politique municipale tente de cerner la portée politique des mobilisations populaires et de cerner l'évolution des positions face à l'action politique municipale. L'organisation du matériel nous oriente vers certaines questions - notamment celle de la signification politique des actions - mais la grande variété des textes et les préoccupations spécifiques des différents auteurs font en sorte que les lignes directrices ne ressortent pas de façon rigide. La richesse du matériel déborde - et de beaucoup - les fils conducteurs.

La même chose est vraie pour le livre d'Ampleman. On commence par des cas concrets pour passer aux outils et finalement à la théorie, l'application des théories de Freire au contexte québécois. Il y a des leçons théoriques derrière cette organisation (il faut baser la théorie sur une compréhension globale et vécue de la réalité) mais il y a aussi une richesse de description qui déroute parfois cette organisation (l'intérêt des études de cas fait que la théorisation semble parfois artificielle et peu liée à la réalité décrite).

La partie la plus intéressante des deux livres est l'interrogation sur le rôle des intellectuels/animateurs/organisateur communautaires/ leaders. Ceci est vrai pour les raisons théoriques (leur rôle pivot qui permet l'intégration des analyses internes et externes) mais aussi des raisons plus pratiques (ce matériel dégage un sens d'implication personnelle, d'interrogation extrêmement vivante, de réflexion qui engage la vie des auteurs). En même temps on peut poser la question à savoir si la préoccupation des auteurs pour l'étude du rôle des intellectuels n'est pas surtout un bon indice de la composition petite-bourgeoise de ces groupes. C'est sans 
aucun doute une question centrale mais l'analyse des actions des groupes l'est aussi.

Ceci nous rappelle que ces deux livres sont plus des collections de textes que des arguments en soi. Ils présentent des documents utiles plus que des analyses théoriques. Ils posent des questions - le rôle des intellectuels, l'importance de la culture dans le changement social, la portée politique des mobilisations - mais ils ne fournissent pas de réponses. Ils sont utiles, finalement, parce qu'ils fournissent un matériel qui permettrait à d'autres d'approfondir nos connaissances sur les groupes populaires au Québec.

Caroline Andrew

Université d'Ottawa 